\title{
Evaluation and improvement of economic efficiency in the sphere of bore-hole drilling methods
}

\author{
Natalia V. Kozlova ${ }^{\text {a }}$, Maria S. Egorova, and Maksim Yu. Safrygin \\ Tomsk Polytechnic University, 634050 Lenin Avenue, 30, Tomsk, Russia
}

\begin{abstract}
One of the important requirements for the methods and technologies, especially in the extractive industry, is cost-effective use of resources. The amount of exploration and industrial bore-hole drilling for various purposes is increasing in today's world. Since drilling is the main type of exploration, it is necessary to improve the technology and to reduce costs. The article discusses an alternative to the existing method of bore-hole drilling developed in Tomsk Polytechnic University on the basis of the discovery made by scientists of the University. Electric pulse drilling technology is an innovation with the estimated base of evidence. The technique consists of rock breaking without drilling in it. This technology is fundamentally different from the existing ones; therefore, its application requires modification and updating of all operation systems. High barriers to entry and introduction to the industry are specific to electric pulse drilling. The authors concluded that the technology of electric pulse drilling provides an alternative of production methods and tools, which allows finding the best option to reduce costs and thereby to increase resource efficiency of production or project.
\end{abstract}

\section{Introduction}

Resources are the basis of industrial production. They form the material composition of the products and provide the manufacturing process. In turn, the products meet the needs of the society. Therefore, well-being of society depends on how physical resources are used and, in particular, how efficient the extraction of natural resources is. Rational and prudent use of natural resources is the true challenge for any industrial company under the conditions of the transition from extensive to intensive economic management.

Economic efficiency of industrial enterprise depends on numerous factors that include either increase in the production effect, for instance, production growth, or cost reduction for the production effect via decrease in labour expenditures and materials consumption.

The decisive factor for economic efficiency is development and introduction of new techniques and technologies developed to meet modern requirements and providing a choice between alternatives of the industrial process. One of the important requirements for the methods and technologies, especially in the extractive industry, is cost-effective use of resources. Primarily, there are the following resources:

- material (both natural and man-made means of production and consumption);

- labor (a combination of physical and intellectual abilities that are used in manufacturing);

- financial (for investment);
- time resources (expended per unit of production) [1]. The transition to resource-saving technologies is impossible without advanced scientific research. The amount of exploration and industrial bore-hole drilling for various purposes is increasing in today's world. Since drilling is the main type of exploration, it is necessary to improve the technology and to reduce costs. It is necessary to take into consideration that the costs of drilling account for a significant part (70 per cent) of the total cost of prospecting and exploration work in the mineral exploration [2].

The article discusses an alternative to the existing method of bore-hole drilling developed in Tomsk Polytechnic University on the basis of the discovery made by the scientists of the University.

\section{Materials and methods}

The most time consuming and expensive operation during drilling is rock destruction. Economic evaluation of exploratory well-drilling depends on the composition (lithological characteristics) of rock, drilling methods and technical equipment. Different methods, primarily mechanical ones, are used for drilling.

A mechanical drilling method has several unconditional advantages. First of all, there is an opportunity to sample rocks for the research and to prepare a geological section. Furthermore, the mechanical method provides the drilling in a given

\footnotetext{
${ }^{\mathrm{a}}$ Corresponding author:knv66@mail.ru
} 
direction, as well as favourable conditions of stripping and studying of groundwater aquifers.

The disadvantages of the mechanical drilling method include:

- wear of expensive operating components of rock cutting equipment, leading to their replacement, especially during the drilling of hard and very hard rocks;

- low energy efficiency that decreases with the increase of the well depth, if the motor is located above the ground. This shortcoming has led to the development of downhole motors (turbo-drills and electric drills), in which an engine is installed in a well above the rock cutting tool; (a combination of physical and intellectual abilities that are used in manufacturing);

- a low rate of penetration.

A particular problem is drilling wells of large diameter (over $300 \mathrm{~mm}$ ). In addition, drilling via boring chisels of any type has a high accident rate. It significantly reduces the performance of drilling operations.

Search for other physical methods of rock destruction during drilling is conducted in Tomsk Polytechnic University. An example of such developments is the electric pulse method of wells drilling. The full name of this method, which was discovered and investigated in TPU, is the electric pulse method for destroying solid dielectric and semiconducting materials. (Certificate for discovery № A-122 of April 29, 1998, with the priority from 14.12.1961 / A.A. Vorobyev, G.A. Vorobyev, A.T. Chepikov "Regularities of solid dielectric breakdown at the interface with the dielectric liquid under the voltage pulse") $[3,4,5]$. The method is based on the discovered effect of the excess of the electric strength of liquid dielectrics and water over the strength of solid dielectrics and rocks at the time before break within 10-6 seconds or less. Consequently, if to place two bipolar electrodes on the surface of a solid body and surround them with a flow medium, in case of the impact of the given voltage impulse, the breakdown occurs inside the solid body. In case of sufficient energy released in the discharged channel, there occurs the explosion with formation of a slabbing crater which is filled with liquid. If the system is a multielectrode the next discharge will occur again in the solid body in a different location. The automatic movement of discharges occurs over the surface of a destructed (bottom hole) until all the areas of the solid body are crushed. After this, a drilling string deepens by means of gravity to the depth of penetration. When this occurs, a drill bit remains stable in the process of destruction, that is a drilling sting or a drill bit does not require rotation as well as mechanical pressure at the bottom hole. It allows increasing the depth significantly, in comparison with mechanical methods, without replacing a bit. This is one more advantage of electropulse drilling [6,7].

A pair of electrodes immersed in liquid with a high specific electrical resistance are used for electric pulse drilling. One electrode is high-voltage, the other is grounded. The electrodes are located at distance $\mathrm{S}$ from each other (Figure 1) and only come in contact with the rock. Thus, there is no load on the drill bit. The drill bit of $270 \mathrm{~mm}$ in diameter is shown in Figure 1. The most important element of the drilling device is a solid insulator [8].

An electric pulse is transmitted between the electrodes. Pulse characteristics are adjusted so that the discharge of electricity passes through inside the rock along the arcuate trajectory and the rock is destroyed over that arc. Rock destruction occurs by detachment of large pieces of rock. Therefore, the method provides high efficiency of energy conversion, low power consumption and a large amount of destruction.

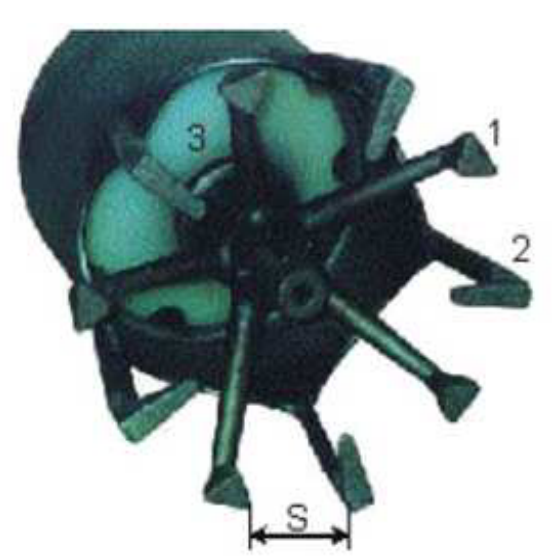

Fig. 1. A drill bit: 1 - high voltage electrode; 2 - grounded electrode; 3 - insulator of a drill bit; $\mathrm{S}$ - the distance between the electrodes

The sequence of electricity discharge introduction in the bottomhole is controlled automatically, eliminating the need for rotation of the drill bit and other parts of the mechanical system. All this allows making the components of the drilling equipment of common steel. Destruction capability of the drill bit is designed for at least $1000 \mathrm{~m}$. The cross-section shape of the well bore may be different depending on the location of electrodes on the drill bit. The range of stress effecting the drill bit determines the possibility of rock disintegration. The discharge voltage value while rock drilling has great dispersion properities. As a result, there is a multifactor interaction of electrophysical properties of a rock and a fluid medium as well as peculiarities of multiple exposure to voltage impulses during rock destruction. It hardly depends on the type of rock. A three-time increase of the distance between electrodes (from 20 to $60 \mathrm{~mm}$ ) leads to 1,6-time increase in the voltage.

This regularity allows making an assumption that drilling holes of big diameters with long electrodes distances $(\mathrm{S}>70 \mathrm{~mm})$ can become the subject of advanced research. A special feature of this method is a significant drilling speed in the setting of a larger diameter of the well bore. Using this method, it is 
possible to destroy virtually any rock, except only the one that has a high electrical conductivity.

Figure 2 shows the simplest view of electric pulse crushing machine for aggregate production. This crusher comprising shell, high voltage and ground electrodes for crushing rock units between them. The crusher shell is filled with dielectric liquid that can be represented by any liquid including process water and a high temperature medium. Ground electrode has through holes which allow fragmented rock of the proper size to move into the storage bin. Finished products are then removed for a subsequent application in concrete

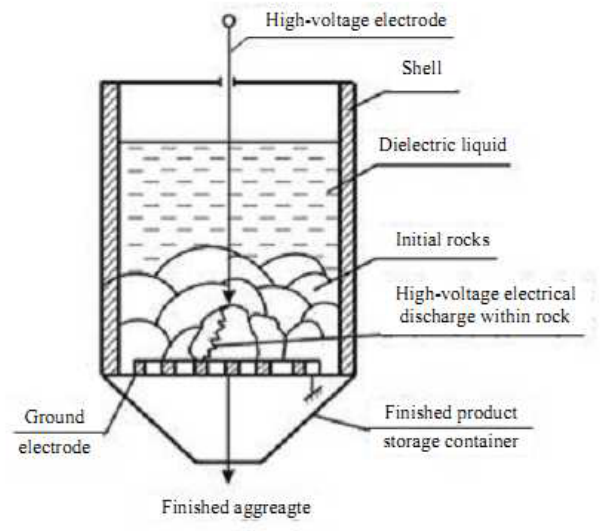

Fig. 2. Electric pulse crusher

Activated aggregate electric pulse technology has a range of characteristics which a known rock grinding does not possess. First, the former implies that crushing is performed by a high voltage electrical discharge produced within rock.

Second, there are no intermediate power transmission member to a stress field of the material destroyed. Moreover, destruction is performed owing to a tensile stress, i.e. the rock unit breaks in tension. While using electric pulse technology selective crushing occurs in places of rock brittleness, heterogeneity, cracking, and cleavage planes. Also, selective coarse crushing occurs in a rock failure zone [9].

The electric-pulse drilling system consists of three major subsystems, such as a drilling device, sources of high voltage surges and a washing system. An important fact is that the constituent components of these systems are produced by an industry and their manufacturing does not require re-establishing of the technical process.

The results of an experiment in rocks destruction via the mechanical and electric pulse method conducted at TPU by Vladislav Fedorovich Vazhov and a group of scientists are considered in the literature. The sample of the drill bit combined with an electrode system for electric pulse destruction and cutters for the mechanical operation of the bottom-hole face were developed. The drill bit diameter was $200 \mathrm{~mm}$. The distance between electrodes was $22 \mathrm{~mm}$. Electric pulse frequency was 2 pulses per second.

The design of the drill bit provides primary rock destruction by electric discharges and secondary rock cutting by mechanical boring tools. The height of the cutter was $6.0 \mathrm{~mm}$. The pressure force onto the drill bit was 7200 N. Deionized water was used as liquid. The oscillograph "Tektronix" recorded the number of electric discharges, discharge inception voltage and current. The hole well of $200 \mathrm{~mm}$ in diameter was formed by mechanical drilling. The depth of the destruction per revolution of the sample amounted to $1 \mathrm{~mm}$ for sandstone, $0.14 \mathrm{~mm}$ for granite. That is 7.14 times less than for the sandstone due to high mechanical performance of granite.

Both mechanical boring and electric pulse drilling were carried out by means of the same samples of sandstone and granite. There was no axial pressure on the drill bit, therefore, the mechanical breakdown of the bottom-hole face was excluded.

Since substantial surface roughness takes place during electric pulse impact on rock, the depth of the destruction was evaluated by the average value, which was $3.6 \mathrm{~mm}$ for a sandstone. That was 3.6 times greater than that after mechanical drilling. The drilling depth for granite was $1.78 \mathrm{~mm}$. That was only 2 times less than for sandstone. There was a 12.7-time increase in the drilling depth.

Combined drilling was conducted on the same samples after mechanical levelling of a surface and under the same conditions as considered above. The depth of combined drilling for the sandstone sample per revolution was $5.7 \mathrm{~mm}$. That was 5.7 times deeper than after mechanical drilling and 1.58 times greater than after electric pulse drilling. The depth of combined drilling for granite was $2.95 \mathrm{~mm}$. That was 1.93 times less than for sandstone. However, in comparison with mechanical drilling of granite, there was a 21.1-time increase in the depth. In comparison with electric pulse drilling there was 1.66-fold of depth increase. This fact is the evidence of insignificant dependence of the electric pulse component of combined drilling on mechanical performance of rocks [10].

\section{Results and discussion}

Well drilling via the electric pulse method includes such basic stages as rock excavation (destruction) and bringing of destructed pieces to the surface. Destroyed rocks are brought to the surface by means of a liquid which is often used for the rocks removal in mines. This liquid must be electrically insulating, however, it may be different in mineral composition, including ordinary water. Thus, the technique consists of rock breaking without drilling in it.

The application area of electric pulse drilling includes drilling of geothermal wells, deep wells for oil and gas, wells under the bridge supports and ventilation shaft wells. The technology of electric pulse drilling can be used in geological surveying and exploration, construction and mining. This method could be useful for drilling of hard and very hard rocks, frozen ground. A pilot installation has been tested in the field 
environment with various rocks in different regions. The equipment demonstrated higher efficiency compared to traditional mechanical methods. This tendency was reflected in such indicators as reduction of time expenditure for the replacement of the drill bit and as a consequence, enhanced productivity of wells drilling.

The main advantages of the electric pulse method of drilling include:

- the conversion of electrical energy of condensators into mechanical work of destruction occurs directly in a destroying material without intermediate stages, providing high performance and low power consumption;

- an opportunity to concentrate high energy at the bottom and to control it depending on drilling conditions;

- a wearproof drill bit, which is not needed to be replaced. This leads to an increase in technical and industrial drilling speed, reduction of operation cost; (a combination of physical and intellectual abilities that are used in manufacturing);

- there is no need to rotate a drillstring and a rockbreaking tool. It eliminates the loss of energy due to friction of the drillstring to the borehole wall. The use of thin-walled (polymer and composite) tubes is possible [11].

Applying the method of electropulse destruction, there is a possibility of achieving higher efficiency in comparison with mechanical ways of destruction with low energy costs. In particular:

- destruction due to mainly pulling (rupture) stress, while rupture strength of hard rocks is sufficiently lower than compression and shearing strength, typical of mechanical methods of rock destruction;

- dynamic loading provides brittle disintegration of rock almost without loss of power and plastic deformation;

- possibility of optimisation of the destruction process which depends on the nature of rock and technical specifications;

- low energy costs are also due to the discharge penetrating depth into rock and its disintegration by rock chipping;

- $\quad$ long-lived drill bits.

The disadvantages of the considered method are work with high voltage (over $200 \mathrm{~kW}$ ), rough walls of the well received as a result of the explosion, as well as large pieces of mud. In addition, the technology of electric pulse drilling is still at the development stage. This technology is fundamentally different from the existing ones; therefore, its employment requires modification and updating of all operation systems. High barriers to entry and introduction to the industry are specific to electric pulse drilling.

This technology is a matter of interest to the different countries. For instance, the opportunity of electric pulse drilling of geothermal wells was considered at the Norwegian University of Science and Technology
(Trondheim). It has been estimated that expenditures would be 100 euros per 1 metre (in 2010 prices) for the well of $6000 \mathrm{~m}$ in depth and about $50 \mathrm{~cm}$ in diameter. Under these conditions, the price of geothermal energy at the mouth of the well will be 1 euro cent per $1 \mathrm{~kW} / \mathrm{h}$. Drilling of such wells via the mechanical method is not effective because of the high cost of produced energy [12].

\section{Conclusion}

A drilling rate is the most important parameter of holes penetration. In case of electropulse drilling, a brand new effect has been obtained: the drilling rate increases along with the increase of the hole diameter at optimisation of drilling conditions (working voltage, distance between electrodes, energy input, cleanout conditions, cutting transport, etc.) The drilling rate increases along with the increase of the distance between electrodes with the same diameter of the drill bit. The drilling rate reduces with the increasing diameter of the drill bit at the fixed distance between electrodes.

It should be concluded that electric pulse drilling technology is an innovation with the estimated base of evidence. This provides an alternative choice of production methods and tools, which allows finding the best option to reduce costs and thereby increase resource efficiency of production or project.

\section{Acknowledgment}

The research was supported by the Strategic Programme of National Research Tomsk Polytechnic University on Competitiveness Enhancement in the Group of Top Level World Research and Academic Institutions.

\section{References}

1. A.A. Dulzon, V.Y. Ushakov, P.S. Chubik, Bull. of TPU, 320 (6), 39 (2012)

2. V.I. Brylin, Drilling of wells for special purposes (P.h. of TPU, Tomsk, 2006)

3. A.A. Vorobiev, Destruction of rocks by electric pulse discharge (P.h. of TSU, Tomsk, 1961)

4. A.B. Uvakov, Impact drilling (Moscow, 1969)

5. V.V. Shtrasser, Investigation of the processes of rocks destruction by hitting balls (Moscow, 1966)

6. V.F. Vazhov, R.R. Gafarov, S.Y. Datskevich, M.Y. Zhurkov, Electric pulse breakdown and destruction of granite (P.h. of TPU, Tomsk, 2009)

7. V.F. Vazhov, R.R. Gafarov, S.Y. Datskevich, M.Y. Zhurkov, V.V. Lopatin, V.M. Muratov, B. Jeffryes, Tech. Phys. Lett., 37 (4), 383 (2011)

8. B.V. Semkin, A.F. Usov, V.I. Kurets, Fundamentals of electric pulse breakdown of materials (Science, St. Petersburg, 1993)

9. V.F. Vazhov, V.M. Muratov, B.S. Levchenko, S.S. Peltsman, D.V. Zhgun, A.V. Adam, J.of Min. Sc., 48 (2), 308 (2012) 
10. V.F. Vazhov, S.Y. Datskevich, M.Y. Zhurkov, V.M. Muratov, B. Jeffryes, Discharge-mechanical method of rock destruction and drilling (P.h. of TPU, Tomsk 2010)

11. Y.I. Protasov, Rocks destruction (Moscow 2001)

12. A. Rodland, Photonics for Harvesting, Proceedings Swissphotonics Workshop, Neuchatel, 125 (2011) 